Rev. Bras. Saúde Prod. Anim., Salvador, v.17, n.2, p.291-298 abr./jun., 2016 http://www.rbspa.ufba.br ISSN 15199940

http://dx.doi.org/10.1590/S1519-99402016000200016

\title{
Indução anestésica do extrato aquoso de cunambí, Clibadium surinamense linn para a realização de biometrias em tambaquis, Colossoma macropomum
}

\author{
Anesthetic induction of the aqueous extract of cunambi, "Clibadium Surinamense" linn \\ to perform biometrics in tambaquis, "Colossoma macropomum"
}
SANTOS, Valéria Amaral dos ${ }^{1 *}$; BRASIL, Paulo Roberto ${ }^{1}$; MALCHER, Cinthia Silva ${ }^{1}$; LOURENÇO, Caio Brito ${ }^{1}$; TRINDADE, Guilherme Vasconcelos ${ }^{1}$; SOUZA, Raimundo Aderson Lobão de ${ }^{1}$

${ }^{1}$ Universidade Federal Rural da Amazônia, Instituto Sócio Ambiental e dos Recursos Hídricos, Belém, Pará, Brasil.

*Endereço para correspondência:valeriaamarals@yahoo.com.br

\section{RESUMO}

A biometria é uma etapa importante do processo de manejo realizada em peixes durante o cultivo. Porem essa pratica expõe os peixes a elevados níveis de estresses que podem ocasionar a debilidade ou ate mesmo a mortalidade dos peixes. O uso de anestésicos naturais como o cunambi, Clibadium surinamense é uma alternativa para minimizar os danos provocados pelo manuseio durante a biometria. O objetivo deste trabalho foi avaliar o cunambí como anestésico para juvenis de tambaqui. Para tanto, foram utilizadas três concentrações de extrato aquoso da planta $(0,5$, $1,5$ e $2,5 \mathrm{ml} / \mathrm{L})$. Foram expostos individualmente 3 peixes $(17,24 \mathrm{~g} \pm 14,01$ e $9,18 \pm 0,91 \mathrm{~cm})$ para cada tratamento e observados o tempo de indução e recuperação anestésica. $\mathrm{O}$ tratamento de $0,5 \mathrm{ml} / \mathrm{L}$ necessitou de maior tempo para atingir o estágio de indução desejado de sedação profunda (7,4 minutos), contudo houve diferença significativa em relação às concentrações de 1,5 e $2,5 \mathrm{ml} / \mathrm{L}$. A concentração de $0,5 \mathrm{ml} / \mathrm{L}$ é a ideal para o procedimento de biometria e breve manejo, pois apesar da demora em induzir o peixe aos diferentes estágios de anestesia, provoca uma rápida recuperação $(8,62$ minutos) em relação a outros tratamentos $1,5 \mathrm{ml} / \mathrm{L}(16,6$ minutos $)$ e $2,5 \mathrm{ml} / \mathrm{L}$ $(23,7 \mathrm{~min})$

Palavras-chave: cunaniol, estresse, manejo

\section{SUMMARY}

Biometrics is an important step in the management process performed in fish during cultivation. However this practice exposes the fish to high levels of stress that can lead to weakness or even fish mortality. The use of natural anesthetic as cunambi, Surinamese Clibadium is an alternative to minimize the damage caused by handling during biometrics. For this we used three aqueous concentrations of plant extract $(0.5,1.5$ and $2.5 \mathrm{ml} / \mathrm{L})$. Individually they were exposed 3 fish $(17,24 \mathrm{~g} \pm$ 14.01 and $9.18 \pm 0,91 \mathrm{~cm}$ ) for each treatment and observed the time of anesthetic induction and recovery. After the anesthesia time the fish were weighed on an analytical balance and placed in aquariums free of the anesthetic solution to return to normal swimming position. After recovery, all subjects were monitored for 96 hours to observe the number of dead and later individuals analysis of optimal concentration. Treatment of $0.5 \mathrm{ml} / \mathrm{L}$ needed more time to reach the desired induction stage of deep sedation ( 7.4 minutes), however there was a significant difference at concentrations of 1.5 and $2.5 \mathrm{ml} / \mathrm{L}$. The concentration of $0.5 \mathrm{ml} / \mathrm{L}$ is ideal for the biometric management procedure and brief, because in spite of the delay in inducing fish to the different stages of anesthesia, causes a rapid recovery (8.62 minutes) in relation to other treatments $1.5 \mathrm{ml} / \mathrm{L}$ (16,6 $\mathrm{min})$ and $2.5 \mathrm{ml} / \mathrm{L}(23.7 \mathrm{~min})$.

Keywords: cunaniol, management, stress 
Rev. Bras. Saúde Prod. Anim., Salvador, v.17, n.2, p.291-298 abr./jun., 2016 http://www.rbspa.ufba.br ISSN 15199940

\section{INTRODUÇÃO}

$\mathrm{Na}$ piscicultura as praticas intensas de manejo provocam uma série de fatores estressantes aos peixes, principalmente aqueles causados durante a biometria, como ferimentos, perda de escamas, estresse e até a morte (VIDAL et al., 2008). O uso de anestésicos é uma alternativa na prática biométrica, pois reduzem o stress e manipulação dos peixes (INOUE et al., 2005; SNEDDON, 2012).

Os anestésicos mais utilizados nas pisciculturas, para as práticas de manejo, são o MS 222, 2-fenoxietanol e a quinaldina. Estas substâncias apresentam um elevado custo, dificultando a aquisição por pequenos produtores. Nesse contexto, a busca por anestésicos de origem natural são importantes, por ser uma alternativa de baixo custo. Os produtos naturais como os óleos essenciais de Lippia alba, Ocimum gratissimum e Aloysia triphylla tem sido estudados devido suas propriedades anestésicas em organimos aquáticos, apresentando resultados promissores (AZAMBUJA et al, 2011; BENOVIT et al, 2012;. PARODI et al, 2012.; SILVA et al., 2012).

O Cunambí (Clibadium surinamense)é uma planta arbórea bastante encontrada na região Norte do Brasil e muito utilizada pela população ribeirinha por facilitar a pesca em rios e igarapés da Amazônia. $O$ uso dessa planta na captura de peixes esta relacionada as suas propriedades toxicas, em que o cunaniol é o principal composto ativo presente em suas folhas.

O tambaqui (Colossoma macropomum) é uma espécie de peixe encontrada largamente na Bacia Amazônica e é uma das mais cultivadas no Brasil e em outros países da América do Sul e Central (FAO, 2014). Devido ao grande potencial do tambaqui na aquicultura, a espécie foi escolhida para o teste com o anestésico natural.

Portanto, devido a carência de estudos referente a utilização de produtos naturais no manejo de tambaqui na região Norte do Pará, este estudo tem como objetivo avaliar a eficácia do extrato aquoso do cunambí como anestésico durante a realização de biometria em juvenis de tambaqui.

\section{MATERIAL E METODOS}

O experimento foi desenvolvido no Laboratório de Aquicultura Tropical da Universidade Federal Rural da Amazônia (UFRA), Belém, Pará, no período de setembro de 2012. Foram utilizados 27 juvenis de tambaqui com peso de $17,24 \mathrm{~g} \pm 14,01$ e comprimento médio de $9,18 \pm 0,91 \mathrm{~cm}$ provenientes da Estação de Piscicultura da SEPAq (Secretária de Estado de Pesca e Aquicultura) localizada no município de Terra Alta, no Estado do Pará.

Os juvenis de tambaqui foram mantidos em caixas de polietileno com capacidade para $150 \mathrm{~L}$ e alimentados duas vezes ao dia com ração comercial (45\% PB) até a fase experimental. A alimentação foi suspensa vinte e quatro horas antes do início do experimento.

Para o preparo do extrato aquoso, as folhas frescas de cunambí foram lavadas com água corrente, secas em papel toalha e cortadas com tesoura em pedaços pequenos para a obtenção $5 \mathrm{~g}$ do material botânico. Em seguida, as folhas foram maceradas com $10 \mathrm{~mL}$ de água para cada $5 \mathrm{~g}$ de folhas cortadas e filtradas com filtro de papel comum. O extrato aquoso foi imediatamente utilizado em aquários com $20 \mathrm{~L}$ de agua. Os juvenis de tambaqui ( $\mathrm{n}=$ 3) foram expostos individualmente a concentrações de $0,5,1,5$, e $2,5 \mathrm{~mL} / \mathrm{L}$ de extrato aquoso de cunambí e observados por dez minutos. 
Rev. Bras. Saúde Prod. Anim., Salvador, v.17, n.2, p.291-298 abr./jun., 2016 http://www.rbspa.ufba.br ISSN 15199940

A fim de avaliar o tempo necessário para atingir os diferentes estágios de indução anestésica e recuperação foi utilizado um cronometro digital. Os diferentes estágios de anestesia foram caracterizados de acordo com Ross \& Ross (2008), conforme apresentado na Tabela 1.

Depois da indução anestésica, os peixes foram pesados em uma balança analítica e transferidos para aquários. Os animais foram monitorados por 96 horas e considerados recuperados até retornarem a posição normal de natação.
Para a análise estatística de cada tratamento (três tratamentos e três repetições) foram analisadas as médias dos tempos de indução e recuperação Foi realizada uma analise de variância (fator único - ANOVA) e quando significativo, foi aplicado o teste de comparação de médias de Tukey $(\mathrm{p}<0,05)$. Para determinar a concentração ideal foi aplicado o teste Student- Newman-Keuls (SNK), através da comparação das medias de cada concentração.

Tabela 1. Estágios de anestesia em peixes*

\begin{tabular}{ccl}
\hline Estágios & Descrição & \multicolumn{1}{c}{ Comportamento } \\
\hline 0 & Normal & $\begin{array}{l}\text { Reativos a estímulos externos; batimentos operculares normais; } \\
\text { reação muscular normal. } \\
\text { Natação agitada, aumento movimento opercular, reativo a estímulos } \\
\text { externos. }\end{array}$ \\
1 & Sedação leve & $\begin{array}{l}\text { Perda parcial equilíbrio e tônus muscular, queda do movimento } \\
\text { opercular. }\end{array}$ \\
2 & Sedação profunda \\
3 & Anestesia profunda & $\begin{array}{l}\text { Perda total equilíbrio e tônus muscular, batimento opercular lento. } \\
\text { Parada da ventilação e morte eventual. }\end{array}$ \\
\hline
\end{tabular}

*Modificada de Ross \& Ross 2008.

\section{RESULTADOS E DISCUSSÃO}

Durante a indução anestésica foram observados os seguintes comportamentos ao primeiro contato com o anestésico: hiperatividade, natação agitada contra a parede do aquário, aumento dos movimentos operculares, natação rápida em curto período, diminuição do ritmo, perda de equilíbrio com o ventre voltado para o lado e reação a estímulos externos com batimento opercular lento. Durante o período experimental não foi registrada nenhuma mortalidade. Como observado em outros estudos utilizando outros anestésicos como eugenol, jambú, também não foram observados mortalidade (INOUE et al.,2011; BARBAS et al., 2016), o que indica que o cunambí possui uma boa margem de segurança durante um breve manejo.
Os dados referentes ao tempo de indução anestésica e de recuperação dos juvenis de tambaqui expostos a diferentes concentrações de extrato aquoso de cunambí encontram-se na Tabela 2.

Nas maiores concentrações (1,5 e 2,5ml), a velocidade das respostas comportamentais foram mais evidentes. Este resultado indica que o comportamento, observado nas maiores concentrações testadas, pode estar relacionado a uma maior concentração do cunaniol, que atua de forma inibitória do sistema GABA (Ácido Gam Aminobutírico)(COSTA et al.,2006). O sistema GABA é o principal neurotransmissor inibitório do sistema nervoso central, pois este causa anestesia e redução dos movimentos respiratórios e dos batimentos cardíacos. (IGGO \& KLEMM, 1996). 
Rev. Bras. Saúde Prod. Anim., Salvador, v.17, n.2, p.291-298 abr./jun., 2016 http://www.rbspa.ufba.br ISSN 15199940

Tabela 2. Tempo médio dos estágios de anestesia e recuperação (em minuto) dos alevinos de tambaqui expostos a diferentes concentrações de extrato aquoso de cunambí. Médias seguidas pela mesma letra em coluna diferem entre si pelo teste de Tukey $(\mathrm{p}<0,05)$

\begin{tabular}{lccccc}
\hline Concentração $(\mathrm{ml} / \mathrm{L})$ & Estágio 1 & Estágio 2 & Estágio 3 & Estágio 4 & Recuperação \\
\hline $0,5 \mathrm{ml}$ & $5,1 \mathrm{a} \pm 2,25$ & $7,4 \mathrm{a} \pm 1,67$ & $*$ & $*$ & $8,62 \mathrm{a} \pm 0,43$ \\
$1,5 \mathrm{ml}$ & $2,2 \mathrm{~b} \pm 0,59$ & $3,5 \mathrm{~b} \pm 1,2$ & $6,3 \mathrm{~b} \pm 1,16$ & $*$ & $16,6 \mathrm{~b} \pm 0,97$ \\
$2,5 \mathrm{ml}$ & $1,2 \mathrm{~b} \pm 1,68$ & $4,0 \mathrm{~b} \pm 1,01$ & $7,8 \mathrm{~b} \pm 1,13$ & $*$ & $23,7 \mathrm{c} \pm 2,72$ \\
\hline
\end{tabular}

*Estagio não alcançado.

Os peixes expostos às três concentrações de extrato aquoso de cunambí não alcançaram todos os estágios de anestesia durante o tempo estimado de 10 minutos. Segundo King et al. (2005), a resistência e tolerância aos anestésicos variam de individuo para individuo, podendo espécies afins diferir muito quanto a utilização de determinado anestésico. No entanto, para o que se pretende não houve a necessidade do aumento das concentrações, pois para a etapa de manejo o estágio 2 (sedação profunda) é o mais adequado, uma vez que o peixe fica imóvel devido a perda parcial de equilíbrio e tônus muscular, facilitando seu manuseio durante todo o processo.

A concentração de $0,5 \mathrm{ml} / \mathrm{L}$ foi $\mathrm{o}$ tratamento que necessitou de maior tempo para atingir o estágio de indução desejado de sedação profunda (Média de 7,4 minutos). Contudo, houve diferença significativa em relação às concentrações de 1,5 e $2,5 \mathrm{ml} / \mathrm{L}$. De acordo com Park et al. (2008) quanto maior a concentração utilizada, menor será o tempo para indução anestesia, ocorrendo uma relação inversa entre esse tempo e a recuperação.

Peixes submetidos às concentrações de 1,5 e $2,5 \mathrm{ml}$ alcançaram até o estagio 3 (anestesia profunda), no entanto o tempo de recuperação foi maior. Contudo, isto pode ser atribuído ao fato de que, quanto maior a concentração de cunambi, maior o tempo necessário à recuperação dos peixes.

Segundo Park et al. (2009), o tempo ideal para levar o peixe a anestesia deve ser menor ou igual a 3 minutos e permitir uma recuperação em um intervalo de tempo máximo de 10 minutos para reduzir os inúmeros impactos ocasionados pelo manejo. No presente trabalho, os resultados encontrados estão acima dos recomendados para a indução anestésica, no entanto a recuperação encontra-se no intervalo sugerido pelos autores citados.

Os resultados relacionados ao tempo de recuperação apresentaram diferença significativa, os peixes anestesiados na concentração de $0,5 \mathrm{ml} / \mathrm{L}$ retornaram mais rápido ( 8 minutos) a posição normal em relação as demais concentrações. Resultado referente a utilização de outros anestésicos de origem natural (eugenol) relataram haver diferença significativa para o tempo de recuperação (PARK et al.,2009).

Nos tratamentos de 1,5 e $2,5 \mathrm{ml} / \mathrm{L}$, verificou-se que em peixes menores, $o$ tempo necessário à indução e recuperação foram maiores. Roubach et al. (2002) utilizando eugenol constataram que em peixes menores a indução anestésica é mais rápida, levando a um maior tempo necessário a recuperação. Olsen et al. (1995), também observaram que a eficácia do anestésico geralmente diminui com o tamanho do peixe, assim em indivíduos menores a indução anestésica é mais lenta do que nos peixes maiores. $\mathrm{O}$ tempo de indução e recuperação encontrase na Figura 2. 
Rev. Bras. Saúde Prod. Anim., Salvador, v.17, n.2, p.291-298 abr./jun., 2016 http://www.rbspa.ufba.br ISSN 15199940

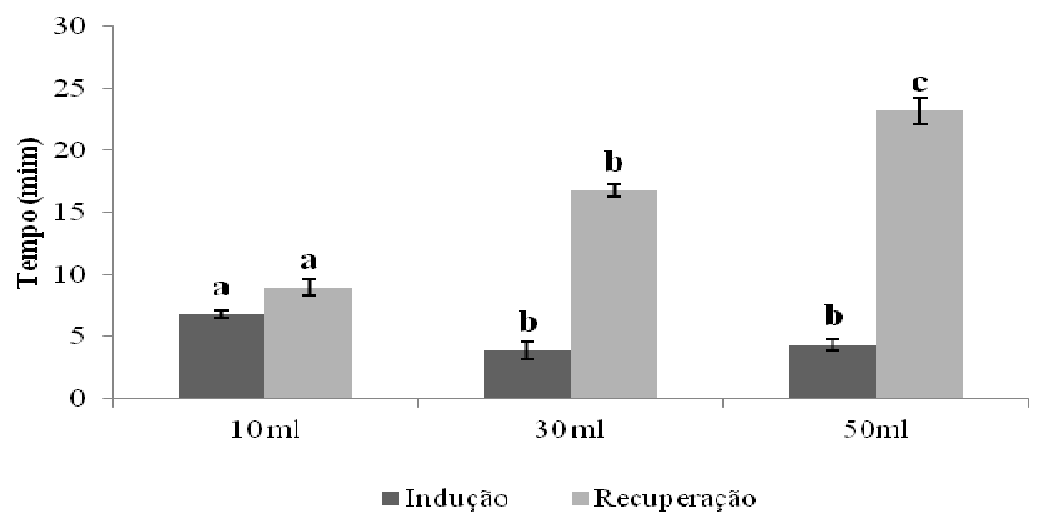

Figura 2. Tempo de indução e recuperação anestésica dos juvenis de tambaqui expostos ao extrato aquoso de cunambí

Embora o cunaniol seja proveniente de uma planta bastante utilizada pela população ribeirinha na Amazônia, ainda não se sabe sobre a existência de resíduos na carcaça e alteração do sabor da carne dos peixes, assim como as alterações fisiológicas dos peixes submetidos ao extrato aquoso de cunambí. Sendo assim, outras pesquisas devem ser realizadas para avaliar o efeito acumulativo ou não do cunaniol. Entretanto, Duke \& Vasquez (1994) relatam que esse princípio ativo é tóxico para humanos. Do mesmo modo, que não há relatos de efeitos tóxicos em humanos que consumiram peixe coletados usando o cunambí, e ambos os consumidores têm consumido peixes coletados desta maneira sem mostrar efeitos imediatos perceptíveis.

Os mecanismos fisiológicos envolvidos na ação anestésica do extrato aquoso de cunambí na indução anestésica do tambaqui ainda não foram completamente entendidos, assim como os efeitos tóxicos resultantes da exposição e manuseio por períodos prolongados ao cunambí.

$\mathrm{O}$ extrato aquoso de cunambí mostrou-se eficaz de acordo com o teste $\mathrm{t}$, para a anestesia de tambaqui, sendo a concentração de $0,5 \mathrm{~m} / \mathrm{L}$ suficiente para a indução anestésica de juvenis durante a biometria.
Os parâmetros físico-químicos oxigênio dissolvido, condutividade e $\mathrm{pH}$ foram significativamente alterados em relação as concentrações testadas, a exceção da temperatura que não houve variação durante $o$ período experimental, mantendo-se dentro da faixa indicada para a espécie.

Entretanto, a estreita variação dos níveis de oxigênio pode ser explicada pela utilização de bastão de vidro para homogeneização da substancia na água dos aquários durante a fase experimental. Contudo, o oxigênio dissolvido estão abaixo do recomendado por Boyd (1990) para o tambaqui, provavelmente esses valores podem estar relacionados a água oriunda de poço artesiano.

$\mathrm{O}$ pH variou de 4,6 a 6,1 ao longo do estudo, podendo ser aceito como adequado ao cultivo de peixes. Para Silva et al. (2007) o Ph ótimo para o bem estar animal esta entre o intervalo entre 6 a 9 .

Não foram registradas mortalidades dos indivíduos pelos baixos níveis de oxigênio e $\mathrm{pH}$, o que confirma que provavelmente os parâmetros não influenciaram no comportamento dos peixes durante a exposição ao anestésico. Os valores médios dos parâmetros físicoquímicos da água obtidos durante $\mathrm{o}$ período experimental encontram-se na Tabela 3. 
Rev. Bras. Saúde Prod. Anim., Salvador, v.17, n.2, p.291-298 abr./jun., 2016 http://www.rbspa.ufba.br ISSN 15199940 http://dx.doi.org/10.1590/S1519-99402016000200016

Tabela 3. Valores médios do oxigênio dissolvido, temperatura, condutividade e pH inicial e final das concentrações utilizadas na biometria. Letras distintas nas linhas indicam diferenças estatisticamente significativas entre os tempos de amostragem pelo teste $\mathrm{t}$ student $(\mathrm{p}>0,05)$

\begin{tabular}{ccccccccc}
\hline Concentrações (g/L) & $\begin{array}{c}\text { ODI } \\
(\mathrm{g} / \mathrm{l})\end{array}$ & $\begin{array}{c}\text { ODF } \\
(\mathrm{mg} / \mathrm{l})\end{array}$ & $\begin{array}{c}\text { TI } \\
\left({ }^{\circ} \mathrm{C}\right)\end{array}$ & $\begin{array}{c}\text { TF } \\
\left({ }^{\circ} \mathrm{C}\right)\end{array}$ & $\begin{array}{c}\text { Cond. i } \\
(\mu \mathrm{S} / \mathrm{cm})\end{array}$ & $\begin{array}{c}\text { Cond. f } \\
(\mu \mathrm{S} / \mathrm{cm})\end{array}$ & pH i & $\mathrm{pH} \mathrm{f}$ \\
\hline $0,5 \mathrm{ml}$ & $1,9^{\mathrm{a}}$ & $2,6^{\mathrm{b}}$ & $28,6^{\mathrm{a}}$ & $28,5^{\mathrm{b}}$ & $0,021^{\mathrm{a}}$ & $0,041^{\mathrm{b}}$ & $4,6^{\mathrm{a}}$ & $5,1^{\mathrm{b}}$ \\
$1,5 \mathrm{ml}$ & $1,7^{\mathrm{a}}$ & $2,7^{\mathrm{b}}$ & $28,6^{\mathrm{a}}$ & $28,6^{\mathrm{b}}$ & $0,022^{\mathrm{a}}$ & $0,081^{\mathrm{b}}$ & $4,6^{\mathrm{a}}$ & $5,7^{\mathrm{b}}$ \\
$2,5 \mathrm{ml}$ & $1,9^{\mathrm{a}}$ & $3,7^{\mathrm{b}}$ & $28,0^{\mathrm{a}}$ & $29,0^{\mathrm{b}}$ & $0,022^{\mathrm{a}}$ & $0,110^{\mathrm{b}}$ & $4,5^{\mathrm{a}}$ & $6,1^{\mathrm{b}}$ \\
\hline
\end{tabular}

O extrato aquoso de cunambi é um eficiente anestésico para juvenis de tambaqui, sendo a concentração de $0,5 \mathrm{ml} / \mathrm{L}$ considerada ideal para a realização de biometria, pois favorece uma rápida recuperação dos animais após o manuseio em relação aos outras concentrações.

\section{REFERENCIAS}

AZAMBUJA, C.R.; MATTIAZZI, J.; RIFFEL, A.P.K;, FINAMOR, I.A.; GARCIA, L.O.; HELDWEIN, C.G.; HEINZMANN, B.M.;

BALDISSEROTTO, B.; PAVANATO, M.A.; LLESUY, S.F. Effect of the essential oil of Lippia alba on oxidative stress parameters in silver catfish (Rhamdia quelen) subjected to transport. Aquaculture, v.319, p.156161, 2011.

BENOVIT, S.C.; GRESSLER, L.T.; SILVA, L.L.; GARCIA, L.O.; OKAMOTO, M.H.; PEDRON, J.S.; SAMPAIO, L.A.; RODRIGUES, R.V. Anesthesia and transport of Brazilian flounder, Paralichthys orbignyanus, with essential oils of Aloysia gratissima and Ocimum gratissimum. Journal of the World Aquaculture Society, v.43, p.896-900, 2012.
BARBAS, L.A.L.; STRINGHETTA, G.R.; GARCIA, L. de O.; FIGUEIREDO, M.R.C.; SAMPAIO, L.A. Jambu, Spilanthes acmella as a novel anaesthetic for juvenile tambaqui, Colossoma macropomum: Secondary stress responses during recovery. Aquaculture, v. 456, p.70-75, 2016.

BOYD, C.E. Water quality in ponds for Aquaculture. Alabama: Brimingham Publishing Co, 1990. 482p.

COSTA, E.A.; ROCHA, F.F.; TORRES, M.L.B.; SOUCCAR, C.; LIMA, T.C.M. de; LAPA, A.J.; LIMA-LANDMAN, M.T.R.. Behavioral effects of a neurotoxic compound isolated from Clibadium surinamense L (Asteraceae).

Neurotoxicology and Teratology, v.28, n.3, p.349-353, 2006.

FOOD AND AGRICULTURE ORGANIZATION OF THE UNITED NATIONS - FAO. State of world fisheries and aquaculture. Roma, Italia, 2014. 243p.

IGGO, A.; KLEMM, W.R. Nervos, sinapses e reflexos. In: SWENSON, M.J.; REECE, W.O. Dukes: fisiologia dos animais domésticos. Rio de Janeiro: Guanabara Koogan, 1996. p.699-713. 
Rev. Bras. Saúde Prod. Anim., Salvador, v.17, n.2, p.291-298 abr./jun., 2016 http://www.rbspa.ufba.br ISSN 15199940 http://dx.doi.org/10.1590/S1519-99402016000200016

INOUE, L.A.K.A.; SANTOS NETO, C. dos; MORAES, G. Efeito do óleo de cravo na resposta de estresse do matrinxã ( Brycon cephalus) submetido ao transporte. Acta Amazônica, v.35, p.289-295, 2005.

KING, W.V.; HOOPER, B.; HILLSGROVE, S.; BENTON, C.; BERLINSKY, D. L. The use of clove oil, metomidate, tricaine methanesulphonate and 2phenoxyethanol for inducing anaesthesia and their effect on the cortisol stress response in black sea bass (Centropristis striata L.). Aquaculture Research, v.36, p.1442- 1449, 2005.

OLSEN, Y.A.; EINARSDOTTIR, L.E.; NILSSEN, K.J. Metomidate anesthesia in Atlantic salmon, Salmo salar, prevents plasma cortisol increase during stress, Aquaculture, v.134, p.155-168, 1995.

PARODI, T.V.; CUNHA, M.A.; HELDWEIN, C.G.; SOUZA, D.M. de; MARTINS, Á.C.; GARCIA, L.O.; WASIELESKY, W.J.R.; MONSERRAT, J.M.; SCHMIDT, D.; CARON, B.O.; HEINZMANN, B.; BALDISSEROTTO, B. The anesthetic efficacy of eugenol and the essential oils of Lippia alba and Aloysia triphylla in post-larvae and sub-adults of Litopenaeus vannamei (Crustacea, penaeidae). Comparative Biochemistry and Physiology, Part C, v.155, n.3, p.462-468, 2012.

PARK, M.O.; HUR, W.J.; IM, S.Y.; SEOL, D.W.; LEE, J.; PARK, In-Seok. Anaesthetic efficacy and physiological responses to clove oil anaesthetized kelp grouper Epinephelus bruneus. Aquaculture Research, v.39, p.877$884,2008$.
PARK, M.O.; IM, S.Y.; SEOL, D.W.; PARK, I.S. Efficacy and physiological responses of rock bream, Oplegnatus fasciatus to anesthetization with clove oil. Aquaculture, v.287, n.3-4, p.427-430, 2009.

QUILLIAM, J.P.; STABLES, R. The effects of cunaniol a polyacetylenic alcohol isolated from the plant Clibadium sylvestre, on piscine behavior. British Journal Pharmacology, v.34, p.679-680, 1968.

ROSS, L.G.; ROSS, B. Anaesthetic and sedative techniques for aquatic Animais, 3nd ed. London, UK: Blackwell , 2009. 222p.

ROSS, L. G.; ROSS, B. Anaesthetic and sedative techniques for aquatic animals. 3rd ed. Oxford: Blackwell Science, 2008.

ROUBACH, R.; GOMES, L.C.; LOURENÇO, J.N.P.; FONSECA, F.A.L; SANTOS, P.J.O.; VAL, A.L. Efficacy of eugenol as anaesthetic for tambaqui juvenile (Colossoma macropomum). In VAL, A.L, ALMEIDA-VAL, V.M.F.; MCKINLEY, D. (Eds) Tropical fish: news and reviews. Vancouver: International Congress on Biology of Fishes, 2002. p.93-96.

SILVA, V.K.; FERREIRA, M.W.; LOGATO, P.V.R. Qualidade da água na Piscicultura. Lavras, MG: Universidade Federal de lavras, 2007. $82 \mathrm{p}$.

SILVA, L.L.; PARODI, T.V.; RECKZIEGEL, P.; GARCIA, V.O.; BÜRGER, M.E.; BALDISSEROTTO, B.; MALMANN, C.A.; PEREIRA, A.M.S.; HEINZMANN, B.M. Essential oil of Ocimum gratissimum L.: anesthetic effect,mechanism of action and tolerance in silver catfish, Rhamdia quelen.

Aquaculture, v.350-353, p.91-97, 2012. 
Rev. Bras. Saúde Prod. Anim., Salvador, v.17, n.2, p.291-298 abr./jun., 2016 http://www.rbspa.ufba.br ISSN 15199940

SNEDDON, L.U. Clinical anesthesia and analgesia in fish. Journal of Exotic Pet Medicine, v.21, p.32-43, 2012.

VIDAL, L.V.O.; ALBINATI, R.C.B.;

ALBINATI, A.C.L.; LIRA, A.D. de;

ALMEIDA, T.R. de; SANTOS, G.B.

Eugenol como anestésico para a tilapia-

do-nilo. Pesquisa Agropecuária

Brasileira, v.43, p.1069-1074, 2008.

FOOD AND DRUG

ADMINISTRATION - US FDA.

Guidance for Industry: concerns

related to the use of closve oil as

anesthetic for fish. Rockville, 2007.

v.1. $355 \mathrm{p}$.

Data de recebimento: 02/04/2015

Data de aprovação: 01/04/2016 